\title{
Erratum to: Risk factors for progression in children and young adults with IgA nephropathy: an analysis of 261 cases from the VALIGA European cohort
}

\author{
Rosanna Coppo $^{1}$ - Danilo Lofaro ${ }^{2} \cdot$ Roberta R. Camilla ${ }^{1} \cdot$ Shubha Bellur $^{3}$ - Daniel Cattran ${ }^{4}$. \\ H. Terence Cook $^{5}$ • Ian S. D. Roberts ${ }^{3} \cdot$ Licia Peruzzi $^{1}$ - Alessandro Amore ${ }^{1}$ - Francesco Emma ${ }^{6}$. \\ Laura Fuiano $^{6}$ - Ulla Berg ${ }^{7} \cdot \operatorname{Rezan}_{\text {Topaloglu }}^{8} \cdot$ Yelda Bilginer $^{8}$ • Loreto Gesualdo $^{9}$ • \\ Rosaria Polci $^{10}$ - Malgorzata Mizerska-Wasiak ${ }^{11}$ - Yasar Caliskan ${ }^{12}$ • Sigrid Lundberg ${ }^{13}$. \\ Giovanni Cancarini $^{14}$ - Colin Geddes ${ }^{15}$ - Jack Wetzels ${ }^{16}$ • Andrzej Wiecek ${ }^{17}$. $^{16}$ \\ Magdalena Durlik $^{18} \cdot$ Stefano Cusinato $^{19} \cdot$ Cristiana Rollino $^{20} \cdot$ Milena Maggio $^{21}$. $^{2}$ \\ Manuel Praga $^{22}$ • Hilde K. Smerud ${ }^{23}$ • Vladimir Tesar ${ }^{24}$ • Dita Maixnerova ${ }^{24}$. \\ Jonathan Barratt $^{25}$ - Teresa Papalia ${ }^{2}$ - Renzo Bonofiglio ${ }^{2}$ - Gianna Mazzucco ${ }^{26}$. \\ Costantinos Giannakakis $^{27}$ • Magnus Soderberg ${ }^{7}$ • Diclehan Orhan ${ }^{8}$ - Anna Maria Di Palma ${ }^{9}$. \\ Jadwiga Maldyk $^{11}$ - Yasemin Ozluk ${ }^{12}$ • Birgitta Sudelin ${ }^{13} \cdot$ Regina Tardanico $^{14}$ David Kipgen $^{15}$. $^{1}$ \\ Eric Steenbergen $^{16}$ • Henryk Karkoszka ${ }^{17}$ - Agnieszka Perkowska-Ptasinska ${ }^{18}$. \\ Franco Ferrario $^{28}$ • Eduardo Gutierrez ${ }^{22}$ - Eva Honsova ${ }^{24}$
}

Published online: 4 October 2016

(C) IPNA 2016

Erratum to: Pediatr Nephrol

DOI: $10.1007 / \mathrm{s} 00467-016-3469-3$

1. The name of the second author, Danilo Lofaro, was rendered incorrectly in the original publication but has now been corrected. 2 . Table 3 in the original publication contains an error introduced in typesetting. The corrected table is reproduced here.

The online version of the original article can be found at http://dx.doi. org/10.1007/s00467-016-3469-3.

Rosanna Coppo

rosanna.coppo@unito.it

Fondazione Ricerca Molinette, Nephrology, Dialysis and Transplantation, Regina Margherita Hospital, Turin, Italy

2 Annunziata Hospital, Cosenza, Italy

3 Oxford University Hospitals, Oxford, UK

4 Toronto General Hospital, University Health Network, Toronto, Ontario, Canada

5 Imperial College, Hammersmith Hospital, London, UK

6 Bambino Gesù Hospital, Rome, Italy

7 Karolinska University Hospital, Huddinge, Sweden

\footnotetext{
8 Hacettepe University, Ankara, Turkey

University of Bari and Foggia, Bari, Italy

10 Belcolle Hospital, Viterbo, Italy

11 University of Warsaw, Warsaw, Poland

12 University of Istanbul, Istanbul, Turkey

13 Karolinska Institutet, Stockholm, Sweden

14 Spedali Civili University Hospital, Brescia, Italy

15 Western Infirmary Glasgow, Glasgow, UK

16 Radboud University, Nijmegen, The Netherlands

17 Silesian University, Katowice, Poland

18 Warsaw Medical University, Warsaw, Poland
} 
Table 3 Correlations between pathological features and outcomes in Cohort 1 and sub-Cohort 2 patients

\begin{tabular}{|c|c|c|c|c|c|c|c|c|}
\hline \multirow{3}{*}{$\begin{array}{l}\text { Pathological } \\
\text { features } \\
\text { included in the } \\
\text { MEST-Oxford } \\
\text { Classification }\end{array}$} & \multicolumn{4}{|c|}{ Cohort $1(n=261)$} & \multicolumn{4}{|c|}{ sub-Cohort $2(n=174)$} \\
\hline & \multicolumn{2}{|c|}{$\begin{array}{l}\text { Rate of renal } \\
\text { function decline }\end{array}$} & \multicolumn{2}{|l|}{$\begin{array}{l}\text { Survival from } \\
\text { combined event }\end{array}$} & \multicolumn{2}{|c|}{$\begin{array}{l}\text { Rate of renal } \\
\text { function decline }\end{array}$} & \multicolumn{2}{|l|}{$\begin{array}{l}\text { Survival from } \\
\text { combined event }\end{array}$} \\
\hline & $\begin{array}{l}\text { Univariate } \\
(\mathrm{ml} / \mathrm{min} / \\
\left.1.73 \mathrm{~m}^{2} / \text { year }\right)\end{array}$ & $\begin{array}{l}\text { Multivariate } \\
\beta(\mathrm{SD})^{\mathrm{b}}\end{array}$ & $\begin{array}{l}\text { Univariate HR } \\
(95 \% \mathrm{CI})\end{array}$ & $\begin{array}{l}\text { Multivariate } \\
\text { HR }(95 \% \mathrm{CI})^{\mathrm{c}}\end{array}$ & $\begin{array}{l}\text { Univariate } \\
(\mathrm{ml} / \mathrm{min} / \\
\left.1.73 \mathrm{~m}^{2} / \text { year }\right)\end{array}$ & $\begin{array}{l}\text { Multivariate } \\
\beta \text { (SD. }^{\mathrm{b}}\end{array}$ & $\begin{array}{l}\text { Univariate HR } \\
(95 \% \mathrm{CI})\end{array}$ & $\begin{array}{l}\text { Multivariate } \\
\mathrm{HR} \\
(95 \% \mathrm{CI})^{\mathrm{c}}\end{array}$ \\
\hline \multicolumn{9}{|c|}{ Mesangial proliferation } \\
\hline M0 & $-1.42 \pm 7.97$ & & 1 & 1 & $-0.85 \pm 7.34$ & & 1 & 1 \\
\hline M1 & $-2.71 \pm 7.32$ & $0.37(0.96)$ & $3.88(1.69-8.88)$ & $2.80(1.21-6.50)$ & $-1.46 \pm 8.3$ & $1.40(1.32)$ & $2.71(0.79-9.35)$ & $1.60(0.35-7.27)$ \\
\hline \multirow{2}{*}{\multicolumn{9}{|c|}{ Segmental glomerulosclerosis }} \\
\hline & & & & & & & & \\
\hline S0 & $-0.89 \pm 5.38$ & & 1 & 1 & $-0.47 \pm 5.07$ & & 1 & 1 \\
\hline S1 & $-2.60 \pm 9.58$ & $-0.29(0.83)$ & $3.51(1.30-9.47)$ & $3.48(1.24-9.76)$ & $-1.68 \pm 9.99$ & $-0.23(1.08)$ & $2.70(0.79-9.27)$ & $2.45(0.61-9.88)$ \\
\hline$p$ & 0.076 & 0.73 & 0.013 & 0.018 & 0.297 & 0.831 & 0.114 & 0.016 \\
\hline \multicolumn{9}{|c|}{ Tubular atrophy/interstitial fibrosis } \\
\hline T0 & $-1.59 \pm 7.53$ & & 1 & 1 & $-0.84 \pm 7.01$ & & 1 & 1 \\
\hline $\mathrm{T} 1-2$ & $-3.83 \pm 10.99$ & $-0.85(1.75$ & $5.31(2.18-12.95)$ & $3.49(1.39-8.76)$ & $-3.06 \pm 13.67$ & $1.81(2.49)$ & $2.86(0.61-13.3)$ & $0.55(0.08-3.88)$ \\
\hline$p$ & 0.242 & 0.627 & $<0.001$ & 0.008 & 0.349 & 0.468 & 0.18 & 0.545 \\
\hline
\end{tabular}

CI, Confidence interval; HR, hazards ratio; eGFR, Estimated glomerular filtration rate

${ }^{a}$ M1, Mesangial score $>0.5$; S1, any segmental sclerosis; T1-2, tubular atrophy and interstitial fibrosis of $>25 \%$. For more detail, see Inclusion criteria, clinical dataset and definitions section and Table 1 footnote

${ }^{\mathrm{b}}$ Multivariate linear models are adjusted for age, gender, initial eGFR, follow-up blood pressure and proteinuria

${ }^{\mathrm{c}}$ Multivariate Cox regression models are adjusted for follow-up blood pressure and proteinuria

19 Borgomanero Hospital, Borgomanero, Italy

20 San Giovanni Bosco Hospital, Turin, Italy

21 Hospital Maggiore di Lodi, Lodi, Italy

22 Hospital 12 de Octubre, Madrid, Spain

23 University of Uppsala, Uppsala, Sweden

24 General University Hospital, Prague, Czech Republic

25 Leicester General Hospital, Leicester, UK

26 University of Turin, Turin, Italy

27 University of Rome, Rome, Italy

28 Monza Hospital, Monza, Italy 